\title{
Research on High-speed Object Detection Using a Planar Capacitive Sensor
}

\author{
Sanmin Shen ${ }^{1,2, a}$, Yong $\mathrm{Ye}^{1, \mathrm{~b}}$, Jiahao Deng ${ }^{1, \mathrm{c}}$ \\ ${ }^{1}$ National Defence Science and Technology Key Laboratory of Mechatronic Engineering and \\ Control, Beijing Institute of Technology, 100086, China \\ ${ }^{2}$ National Key Laboratory of Science and Technology on Electronic Test and Measurement, North \\ University of China, 030051, China \\ aemail:shensanming@nuc.edu.cn, bemail:yong304256381@163.com, cemail:bitdjh@bit.edu.cn
}

Keywords: High-speed object detection; Planar capacitive sensor; Quasi-electrostatic field

\begin{abstract}
A new method of high-speed object detection using planar capacitive sensor was presented. The planar capacitive consists of two electrodes. A sine signal was applied on one electrode to form a quasi-electrostatic field and another electrode was use to detect the object signal. The voltage of the detecting electrode is changed when an object approached to the planar capacitive. The detection model of the planar capacitive sensor was established. The relationship between the rendezvous distance and the voltage variation was deduced. The increase of the electrode diameter and the decrease of the electrodes space contributed to the improvement of the capacitive sensor's sensitivity. The experimental results showed that the planar capacitive sensor could achieve short range detection for high-speed object.
\end{abstract}

\section{Introduction}

The planar capacitive sensors has been developed in a wide range of applications for decades because of its simplicity, low power consumption and high sensitivity [1]. In 2004, Jian Z. Chen et al. presented a miniaturized coplanar capacitive sensor for detecting droplet position, volume, composition and mass loss for volumes in the nanoliter range [2]. In 2007, N.Kirchner et al. introduced an adaptive capacitive sensor for obstacle ranging. The range of detect distance was up to 500mm [3]. In 2010, PH.D Li Nan discussed the sensitivity difference between three types of ECT sensor which is including square-shaped, octagon-shaped and traditional circular-shaped ECT sensor structures [4]. In 2011, Caglar Elbuken et al. presented the means to detect the presence, size and speed of micro-droplets in microfluidic devices using commercially available capacitive sensors [5]. Under the background of interception system of tank armor, this paper present a new high-speed moving object detected method using a planar capacitive sensor.

\section{Model analysis of the planar capacitive detection}

The planar capacitive sensor consists of a planar capacitor, a voltage detection circuit, a plexiglass block, a metallic box and a signal processing circuit. The planar capacitive has two electrodes - a driving electrode and a sensing electrode. Coplanar electrodes which made by thin copper are used to form a capacitance. A voltage detection circuit includes an oscillation circuit and a rectifier circuit. The oscillation circuit is a Clapp oscillator used as an excitation of the driving electrode. It creates a sine signal. The sine signal is applied on the driving electrode to form a quasi-electrostatic field. The voltage of the sensing electrode is changed when the object approaches to the planar capacitive as shown in Fig.1. The planar capacitive is pasted on a rectangular plexiglass. The plexiglass is fixed on a metallic box which is connected to signal ground. The metallic box has the same shape with the plexiglass block as shown in Fig.2.

To simplify the problem, we make the following assumptions: I. All of the free charges focus on the center of the electrodes surface. II. The charges in the object were regarded as a point charges.Suppose that $\mathrm{T}$ is in any point upon $\mathrm{A}$ and $\mathrm{B}$. We define the attitude angle $\alpha$ is positive 


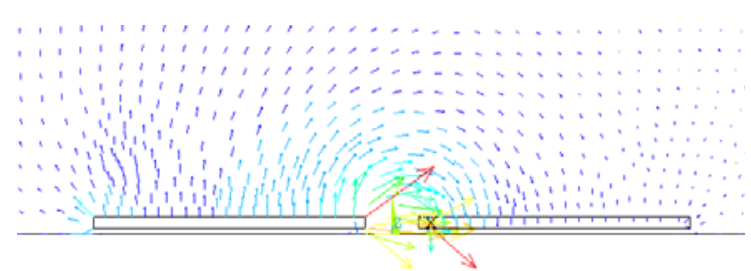

(a) Without a object

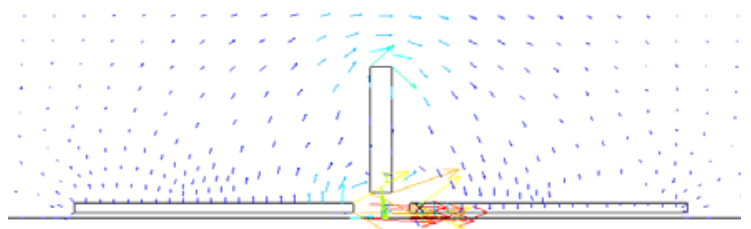

(b) With a object

Fig. 1 Schematic view of electric field lines of the planar electrodes

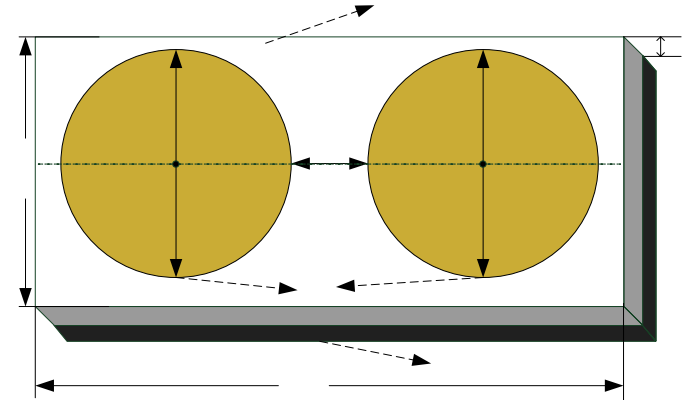

Fig.2 Schematic view of the planar capacitive sensor

when TA and TO (the vertical line) are at a anti clockwise angle or is negative (as shown in Fig.3). It is opposed as $\beta$.

Suppose that $\mathrm{T}$ is in any point upon $\mathrm{A}$ and $\mathrm{B}$. We define the attitude angle $\alpha$ is positive when TA and TO (the vertical line) are at a anti clockwise angle or is negative (as shown in Fig.3). It is opposed as $\beta$.

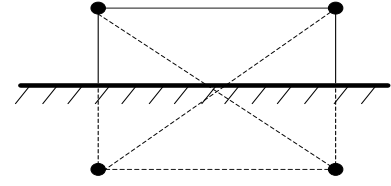

(a) without a object

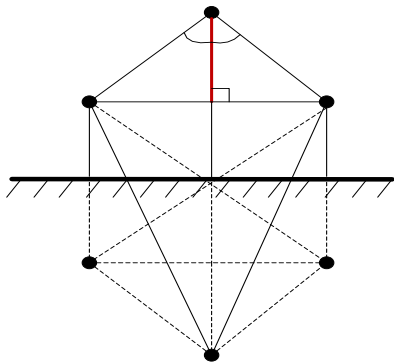

(b) with a object

Fig.3 Charge pattern of the planar capacitive sensor

The voltage between $\mathrm{A}$ and $\mathrm{B}$ without a moving object is given as:

$$
U_{a b}=\Phi_{a}-\Phi_{b}
$$

Where $\Phi_{\mathrm{a}}$ is the electric potential of electrode $\mathrm{A} ; \Phi_{\mathrm{b}}$ is the electric potential of electrode $\mathrm{B}$; The charge pattern of the planar capacitive sensor with a moving object based on mirror method is shown in Fig.3. The equivalent electric potential of electrode $\mathrm{A}$ and $\mathrm{B}$ according to the superposition principle can be written as:

$$
\begin{aligned}
& \varphi_{a}=\Phi_{a}+\frac{Q_{t}}{4 \pi \varepsilon_{e} h / \cos \alpha}-\frac{Q_{t}}{4 \pi \varepsilon_{e} \sqrt{(h \tan \alpha)^{2}+(2 t+h)^{2}}} . \\
& \varphi_{b}=\Phi_{b}+\frac{Q_{t}}{4 \pi \varepsilon_{e} h / \cos \beta}-\frac{Q_{t}}{4 \pi \varepsilon_{e} \sqrt{(h \tan \beta)^{2}+(2 t+h)^{2}}} .
\end{aligned}
$$

With the object existence, the Eq.1 can be changes as: 


$$
\begin{aligned}
u_{a b}= & \varphi_{a}-\varphi_{b} \\
= & U_{a b}+\frac{Q_{t}}{4 \pi \varepsilon_{e}}\left[\frac{1}{h / \cos \alpha}-\frac{1}{\sqrt{(h \tan \alpha)^{2}+(2 t+h)^{2}}}-\frac{1}{h / \cos \beta}\right. \\
& \left.+\frac{1}{\sqrt{(h \tan \beta)^{2}+(2 t+h)^{2}}}\right] .
\end{aligned}
$$

Subtracting the Eq.4 and the Eq.1, we can find:

$$
\begin{aligned}
\Delta U_{a b} & =u_{a b}-U_{a b}=\frac{Q_{t}}{4 \pi \varepsilon_{e}}\left[\frac{1}{h / \cos \alpha}-\frac{1}{\sqrt{(h \tan \alpha)^{2}+(2 t+h)^{2}}}-\frac{1}{h / \cos \beta}+\frac{1}{\sqrt{(h \tan \beta)^{2}+(2 t+h)^{2}}}\right] \\
& =\frac{Q_{t}}{4 \pi \varepsilon_{e}}\left[\frac{1}{h / \cos \alpha}\left(1-\frac{1}{\sqrt{\sin ^{2} \alpha+\frac{(2 t+h)^{2}}{h^{2}} \cos ^{2} \alpha}}\right)\right] \\
& =\frac{Q_{t}}{4 \pi \varepsilon_{e}}\left[\frac{\cos \alpha}{h}\left(1-\frac{1}{\sqrt{1+\frac{4 t^{2}+4 h t}{h^{2}} \cos ^{2} \alpha}}\right)-\frac{\cos \beta}{h}\left(1-\frac{1}{\sqrt{\sin ^{2} \alpha+\frac{(2 t+h)^{2}}{h^{2}} \cos ^{2} \alpha}}\right)\right]
\end{aligned}
$$

When $t<<h$, the inequalities $\frac{4 t^{2}+4 h t}{h^{2}} \cos ^{2} \alpha<1$ and $\frac{4 t^{2}+4 h t}{h^{2}} \cos ^{2} \beta<1$ are correct. So with Taylor expansion the Eq.5 can be written as:

$$
\begin{aligned}
\Delta U_{a b}= & \frac{Q_{t}}{4 \pi \varepsilon_{e}}\left[\frac{\cos \alpha}{h}\left(1-\frac{1}{\sqrt{1+\frac{4 t^{2}+4 h t}{h^{2}} \cos ^{2} \alpha}}\right)-\frac{\cos \beta}{h}\left(1-\frac{1}{\sqrt{1+\frac{4 t^{2}+4 h t}{h^{2}} \cos ^{2} \beta}}\right)\right] \\
= & \frac{Q_{t}}{4 \pi \varepsilon_{e}}\left\{\frac{\cos \alpha}{h}\left\{1-\left[1-\frac{1}{2} \frac{4 t^{2}+4 h t}{h^{2}} \cos ^{2} \alpha+\frac{3}{8}\left(\frac{4 t^{2}+4 h t}{h^{2}}\right)^{2} \cos ^{4} \alpha \cdots\right]\right\}-\right. \\
& \left.\frac{\cos \beta}{h}\left\{1-\left[1-\frac{1}{2} \frac{4 t^{2}+4 h t}{h^{2}} \cos ^{2} \beta+\frac{3}{8}\left(\frac{4 t^{2}+4 h t}{h^{2}}\right)^{2} \cos ^{4} \beta \cdots\right]\right\}\right\} \\
= & \frac{Q_{t}}{4 \pi \varepsilon_{e}}\left[\frac{1}{2} \frac{4 t^{2}+4 h t}{h^{3}}\left(\cos ^{3} \alpha-\cos ^{3} \beta\right)-\frac{3}{2}\left(\frac{t^{2}+h t}{h^{2}}\right)^{2} \frac{\cos ^{5} \alpha-\cos ^{5} \beta}{h} \cdots\right] .
\end{aligned}
$$

If the 4-order power and the higher order power of $r^{-1}$ are ignored, the Eq.6 is as follows:

$$
\Delta U_{a b}=\frac{Q_{t}}{8 \pi \varepsilon_{e}} \frac{\left(4 t^{2}+4 h t\right)\left(\cos ^{3} \alpha-\cos ^{3} \beta\right)-3 t^{2}\left(\cos ^{5} \alpha-\cos ^{5} \beta\right)}{h^{3}} .
$$

From the Eq.7, a fact is shown that the $U_{a b}$ decreases with the $h$ increasing and the decrease is inversely proportional to $h^{3}$.

The equivalent capacitance between two electrodes can be computed using Gaussian theorem. According to the Laplace equation, we can find:

$$
\vec{E}=-\nabla U=-\left(\frac{\partial U}{\partial r} \overrightarrow{e_{r}}+\frac{1}{r} \frac{\partial U}{\partial \varphi} \overrightarrow{e_{\varphi}}\right)=-\frac{U}{r \pi} \overrightarrow{e_{\varphi}}
$$




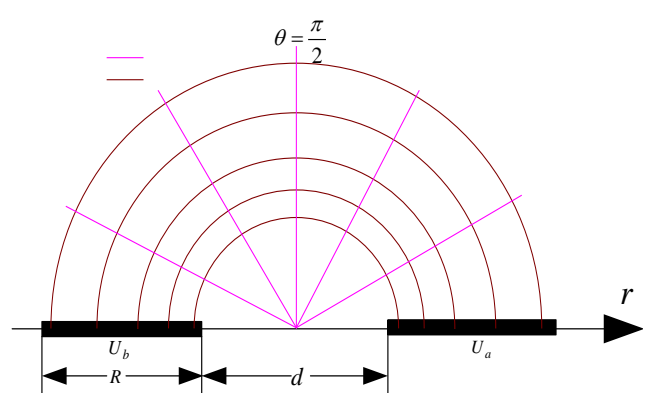

Fig.4 Equipotential surfaces and electric flux lines of the planar capacitive The surface charge density is as follows:

$$
\sigma=\left|\varepsilon_{e} \vec{E}\right|=\frac{U_{a b}}{r \pi} \varepsilon_{e}
$$

The integral surface in two-dimensional coordinate system is shown in Fig.5. The charge of the electrode can be calculated as follows:

$$
\begin{aligned}
Q & =\oiint \varepsilon_{e} E \cdot d s=\int_{d / 2}^{\frac{(d+R)}{2}} \frac{U_{a b}}{r \pi} \varepsilon_{e} \cdot d r \int_{0}^{2 R} 2 \sqrt{R^{2}-\left(R-r+\frac{d}{2}\right)^{2}} \cdot d z+\int_{\frac{(d+R)}{2} \frac{\frac{d}{2}+R}{r \pi} \frac{U_{a b}}{r \pi} \varepsilon_{e} \cdot d r \int_{0}^{2 R} 2 \sqrt{R^{2}-\left(r-\frac{d}{2}-R\right)^{2}} \cdot d z} \\
& =\int_{-R}^{R} \frac{U_{a b}}{\left(r+R+\frac{d}{2}\right) \pi} \varepsilon_{e} \cdot d r \int_{0}^{2 R} 2 \sqrt{R^{2}-r^{2}} \cdot d z \approx U_{a b} R \varepsilon_{e}\left[2 R+d-\sqrt{4 R d+d^{2}}\right]
\end{aligned}
$$

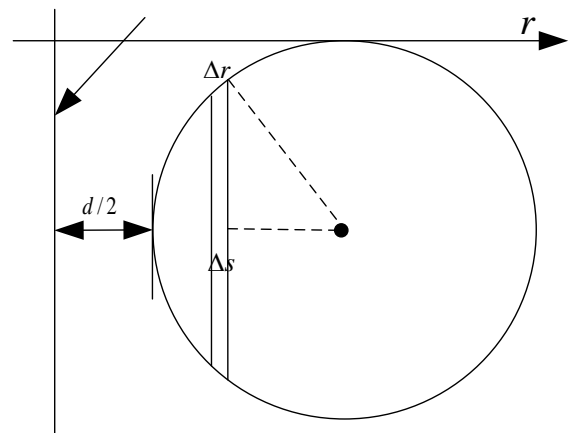

Fig.5 Integral surface in two-dimensional coordinate system.

An approximate solution of the Eq.10 is given using Maple. Thus the capacitance created by the two electrodes can be calculated as

$$
C_{a b}=Q / U_{a b}=R \varepsilon_{e}\left[2 R+d-\sqrt{4 R d+d^{2}}\right]
$$

According to the Eq.11, the partial derivative of the electrode spacing and the diameter with respect to $C_{a b}$ are given as follows:

$$
\left\{\begin{array}{l}
\frac{\partial C_{a b}}{\partial R}=\varepsilon_{e}\left[4 R+d-\frac{6 R d+d^{2}}{\sqrt{4 R d+d^{2}}}\right]>0 \\
\frac{\partial C_{a b}}{\partial d}=R \varepsilon_{e}\left[1-\frac{2 R+d}{\sqrt{4 R d+d^{2}}}\right]=R \varepsilon_{e}\left[1-\frac{\sqrt{4 R^{2}+4 R d+d^{2}}}{\sqrt{4 R d+d^{2}}}\right]<0
\end{array} .\right.
$$

Then $C_{a b}$ increases with $R$ and decreases with $d$. The $\Delta U_{o}$ has the same relationship with $R$ and $d$. 


\section{Experimental results and discussion}

The physical map of the planar capacitive sensor is shown in Fig 6. The length of the sensor is $L=400 \mathrm{~mm}$, the width is $W=255 \mathrm{~mm}$. The thickness of the plexiglass block is $T=2 \mathrm{~mm}$. The oscillation amplitude of the oscillation circuit is $100 \sim 110 \mathrm{~V}$ and the oscillation frequency is $2.3 \mathrm{MHz}$. Three types of shells were used in the experiment. They are 105-High-Explosive Anti-Tank (the maximum diameter is $105 \mathrm{~mm}$, the length is $720 \mathrm{~mm}$ ), $\Phi 25$-kinetic energy projectile (the maximum diameter is $25 \mathrm{~mm}$, the length is $950 \mathrm{~mm}$ ) and 40 -HEAT (the maximum diameter is $40 \mathrm{~mm}$, the length is $900 \mathrm{~mm}$ ). These three types of shells are numbered as S1,S2 and S3.

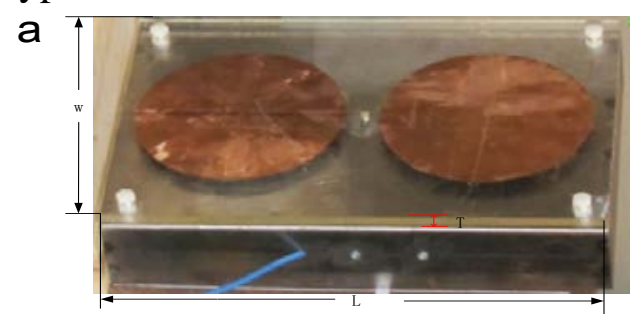

Fig.6 The photo of the planar capacitive sensor

The static experimental results of S1 with different diameter of the electrodes is shown as Fig 7(a). The results with different of electrode spacing is shown as Fig 7(b). The larger the electrodes are, the higher the voltage variation is. And the smaller the electrodes spacing are, the higher the voltage variation is.

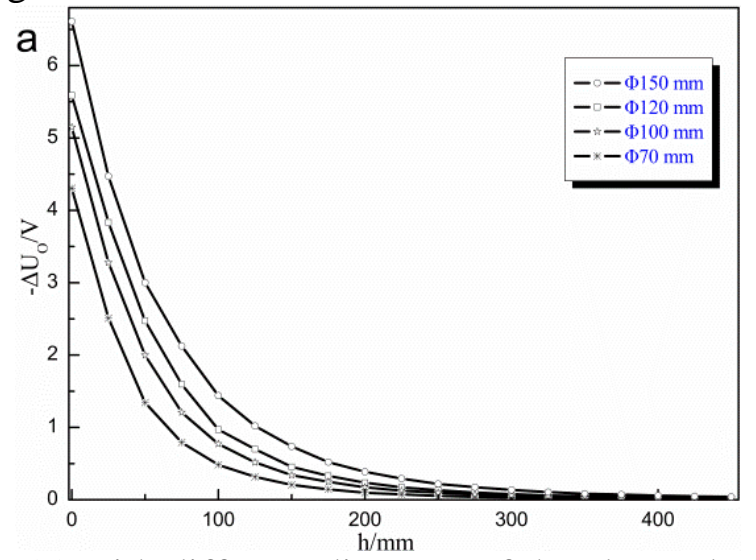

(a) with different diameter of the electrodes

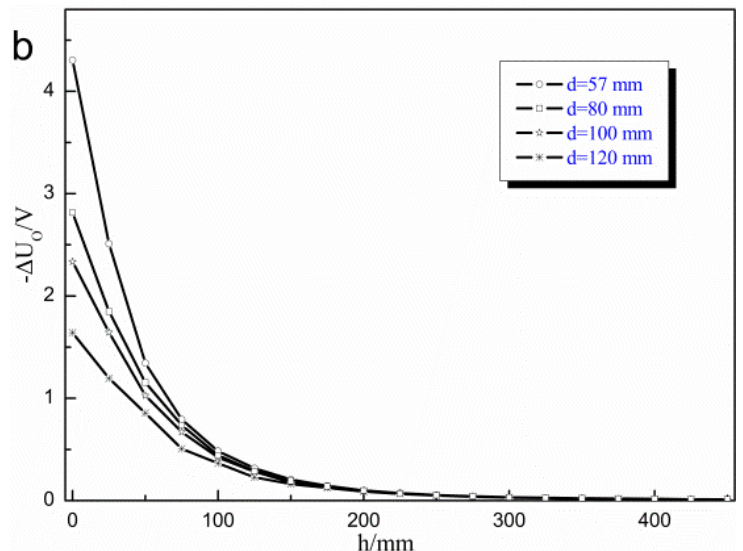

(b) with different electrode spacing.

Fig.7 Comparison of detection voltage change

A numerous measure using 150-mm-diameter electrodes were carried out for the attack angle $\gamma=90^{\circ}$ with the object moving along the axis of the center point of the planar capacitive sensor with a level shift. We define $h$ is rendezvous distance. When $h>40 \mathrm{~mm}$, the voltage variation $\left(\Delta U_{o}\right)$ is obviously inversely proportional to $h^{3}$ (see Fig.5), which proves the correctness of the theoretical model. The error bars show that the smaller of the $h$ is, the larger the sample error is . We can set a threshold to start the recognition program. As shown in Fig.8(a), the threshold is 0.05V when $h>40 \mathrm{~mm}$. Fig.8(b) shows the results of the ratio of detection voltage variation sum between every adjacent $50 \mathrm{~mm}$. The curve is approximately linear. The ratio of 0 to $50 \mathrm{~mm}$ is $2.0 \sim 2.1$, the ratio of the following adjacent $50 \mathrm{~mm}$ is $1.75 \sim 2.0,1.5-1.7,1.4 \sim 1.5$ and $1.2 \sim 1.4$ respectively. The detection distance can be obtained by the ratio. The results of dynamic experiments with three types of shells are shown in Fig.8(c). The design of dynamic experiments is that the explosive device is exploded when the rendezvous distance is $200 \mathrm{~mm}$. The speed of three types of shells is $1080 \mathrm{~m} / \mathrm{s}, 1600 \mathrm{~m} / \mathrm{s}$ and $300 \mathrm{~m} / \mathrm{s}$ respectively. Using the method which combined threshold with ratio, the actual detection distances are $160 \mathrm{~mm}, 170 \mathrm{~mm}$ and $220 \mathrm{~mm}$ respectively. The error of distance measurement is in the range of $100 \mathrm{~mm}$. 


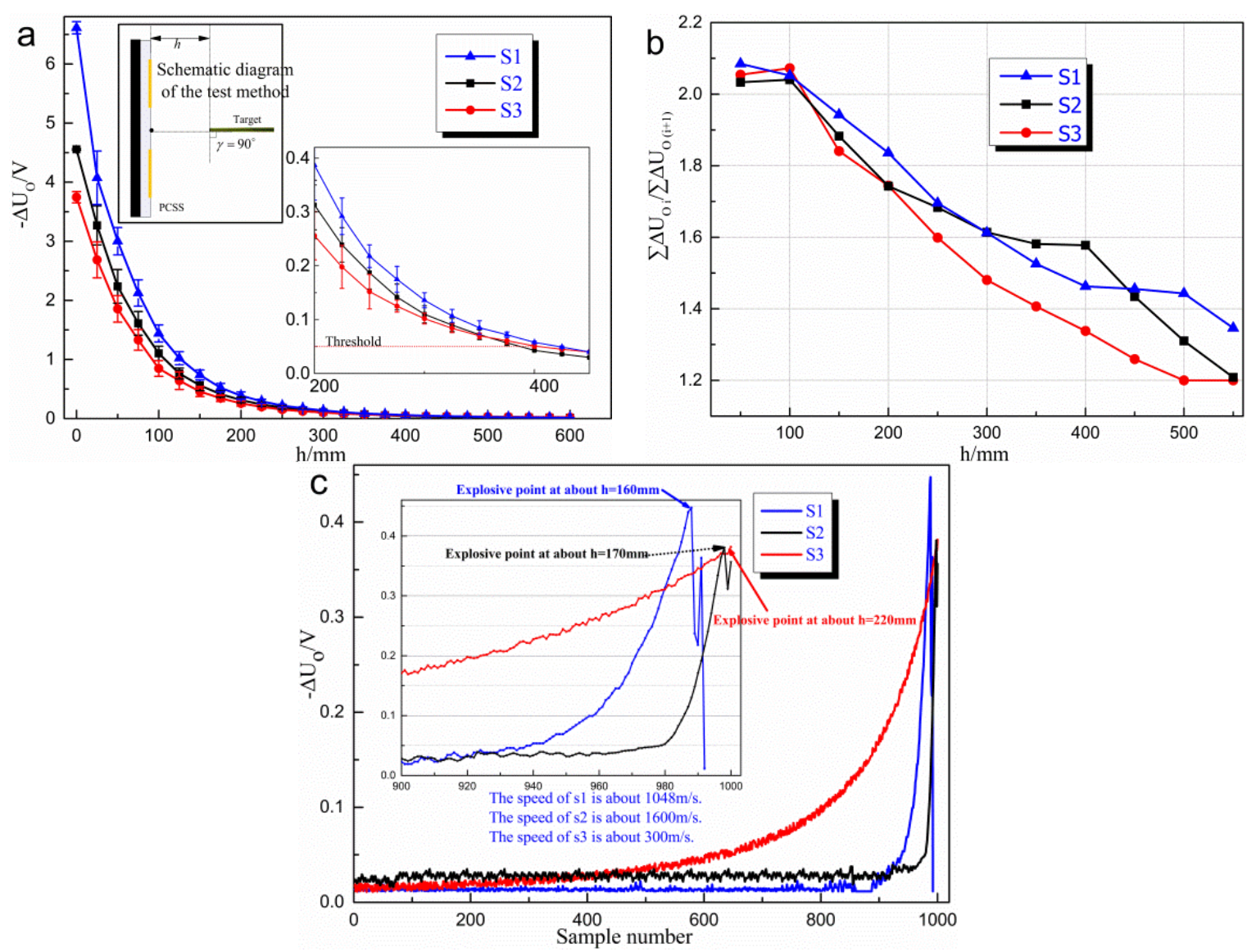

Fig.8 Comparison of detection voltage variation (a) and ratio (b) with three types of shells (c)

\section{Conclusion}

A new high-speed object detection using a planar capacitance sensor was presented. And the theoretical model of the system was established. The increase of the electrode diameter and the decrease of electrode spacing contributed to the improvement of the capacitive sensor's sensitivity. The theoretical analysis and experimental results showed that the planar capacitive sensor could achieve short range detection for high-speed object with real-time performance and high accuracy. Compared with the radar and laser detection system, the method has the advantages of anti jamming, dust environment performance, simple structure and low cost.

\section{References}

[1] X.Li, Low-cost smart capacitive sensor for position and speed measurement, Ph.D. dissertation, Delft Univ. Technol, The Netherlands, May 1997.

[2] N.Kirchner, D.K. Liu, T.Taha, G.Paul. Capacitive object ranging and material type classifying sensor, C. The Eighth International Conference on Intelligent Technologies (InTech'07), Sydney, Australia, 2007,pp.130-135.

[3] M.F.A Rahman, A.A Manaf, M.R Arshad. Capacitive effect of coplanar electrodes partially outside the microchannel region for underwater microfluidic-based sensor, J. Indian Journal of Geo-Marine Sciences, 2013,8(42), pp.987-991.

[4] LI Nan. Research on proximity capacitive sensor design and ECT technique, Ph.D. dissertation, Xian, Xidian University,2010.

[5] Caglar Elbuken, Tomasz Glawdel, Danny Chan, Carolyn L. Ren. Detection of microdroplet size and speed using capacitive sensors, J. Sensors and Actuators A: Physical, 2011, 171, pp.55-62. 